Supporting Information

\title{
General Synthesis of Thiophene and Selenophene-Based Heteroacenes
}

\author{
Toshihiro Okamoto, Kenichi Kudoh, Atsushi Wakamiya, and Shigehiro Yamaguchi* \\ Department of Chemistry, Graduate School of Science, Nagoya University, and SORST, Japan \\ Science and Technology Agency (JST), Chikusa, Nagoya 464-8602, Japan
}

\section{Experimental Section}

General. Melting point (mp) determination was performed using a Yanaco MP-S3 instrument. ${ }^{1} \mathrm{H}$ and ${ }^{13} \mathrm{C}$ NMR spectra were measured with a JEOL EX-270 spectrometer, a JEOL A-400 spectrometer, or a VARIAN INOVA-500 spectrometer. IR spectra were measured with a JASCO FTIR 430 spectrometer. Cyclic voltammograms were recorded on a CHI600A instrument. Thin layer chromatography (TLC) was performed on plates coated with $0.25 \mathrm{~mm}$ thick silica gel 60F-254 (Merck) or $0.25 \mathrm{~mm}$ thick aluminum oxide 60F-254 (Merck). Column chromatography was performed using a Fuji Silysia silica gel PSQ60B $(60 \mu \mathrm{m})$. The starting material $\mathbf{1} \mathbf{a}^{1}$ and compounds $\mathbf{2 a}$ and $\mathbf{3} \mathbf{a}^{2}$ are the literature known compounds. Copper nanopowder was purchased from Aldrich. All reactions were carried out under argon atmosphere except where noted.

1,4-Bis(o-bromophenyl)diacetylene (1a). To a suspension of copper iodide (1.67 mmol, $319 \mathrm{mg})$ and $0.50 \mathrm{~mL}$ of $N, N, N^{\prime}, N$ '-tetramethylethylenediamine $(3.35 \mathrm{mmol})$ in $35 \mathrm{~mL}$ of acetone was added $o$-bromophenylacetylene $(6.06 \mathrm{~g}, 33.5 \mathrm{mmol})$. The mixture was stirred at room temperature for $1 \mathrm{~h}$ while oxygen gas was bubbled through the solution. After removal of the solvent under reduced pressure, $1 \mathrm{~N} \mathrm{HCl}$ aqueous solution was added and the mixture was extracted with $\mathrm{CH}_{2} \mathrm{Cl}_{2}$. The organic layer was washed with water and brine, dried over $\mathrm{MgSO}_{4}$, filtered, and concentrated under reduced pressure. The obtained solid was further washed with hexane to give pure 1a $(5.21 \mathrm{~g}, 14.5 \mathrm{mmol})$ in $86 \%$ yield: ${ }^{1} \mathrm{H}$ NMR (400 MHz, $\left.\mathrm{CDCl}_{3}\right): \delta$ 7.62-7.57 (m, 4H), 7.32-7.21 (m, 4H).

1,4-Bis(2-bromo-4-anisyl)diacetylene (1c). This compound was prepared from (2-bromo4-anisyl)acetylene essentially in the same manner as described for 1a in 94\% yield as white solid: mp. $145-147{ }^{\circ} \mathrm{C} . \quad{ }^{1} \mathrm{H}$ NMR $\left(400 \mathrm{MHz}, \mathrm{CDCl}_{3}\right): \delta 7.48(\mathrm{~d}, J=8.8 \mathrm{~Hz}, 2 \mathrm{H}), 7.13(\mathrm{~d}, J=$ $2.4 \mathrm{~Hz}, 2 \mathrm{H}), 6.82(\mathrm{dd}, J=8.8,2.4 \mathrm{~Hz}, 2 \mathrm{H}), 3.82(\mathrm{~s}, 6 \mathrm{H}) .{ }^{13} \mathrm{C} \mathrm{NMR}\left(67.5 \mathrm{MHz}, \mathrm{CDCl}_{3}\right)$ : $\delta 160.35,135.16,126.99,117.92,116.25,113.61,80.64,76.70,55.71$. Anal. Calcd for $\mathrm{C}_{18} \mathrm{H}_{12} \mathrm{Br}_{2} \mathrm{O}_{2}: \mathrm{C}, 51.46 ; \mathrm{H}, 2.88$. Found: C, 51.65; H, 2.83. 
1,4-Bis(2-bromo-4-trifluoromethylphenyl)diacetylene (1d). This compound was prepared from (2-bromo-4-trifluoromethylphenyl)acetylene essentially in the same manner as described for 1a in $92 \%$ yield as white solid: mp. $90-91{ }^{\circ} \mathrm{C} .{ }^{1} \mathrm{H}$ NMR $\left(400 \mathrm{MHz}, \mathrm{CDCl}_{3}\right.$ ): $\delta 7.88$ (s, $2 \mathrm{H}), 7.82(\mathrm{~d}, J=8.0 \mathrm{~Hz}, 2 \mathrm{H}), 7.57(\mathrm{~d}, J=8.0 \mathrm{~Hz}, 2 \mathrm{H}) .{ }^{13} \mathrm{C} \mathrm{NMR}\left(100 \mathrm{MHz}, \mathrm{CDCl}_{3}\right)$ : $\delta 134.73,132.06\left(\mathrm{q}, J_{\mathrm{C}-\mathrm{F}}=33.3 \mathrm{~Hz}\right), 129.62\left(\mathrm{q}, J_{\mathrm{C}-\mathrm{F}}=4.1 \mathrm{~Hz}\right), 127.48,126.55,124.08,124.04$ $\left(\mathrm{q}, J_{\mathrm{C}-\mathrm{F}}=4.1 \mathrm{~Hz}\right), 80.81,79.55$. Anal. Calcd for $\mathrm{C}_{18} \mathrm{H}_{6} \mathrm{Br}_{2} \mathrm{~F}_{6}: \mathrm{C}, 43.58 ; \mathrm{H}, 1.22$. Found: $\mathrm{C}$, $43.76 ; \mathrm{H}, 1.16$.

1,4-Bis(3-bromo-5-trimethylsilyl-2-thienyl)diacetylene (1e). This compound was prepared from (3-bromo-5-trimethylsilyl-2-thienyl)acetylene essentially in the same manner as described for 1a in 73\% yield as white solid: mp. $122-124{ }^{\circ} \mathrm{C} .{ }^{1} \mathrm{H}$ NMR $(500 \mathrm{MHz}$, $\left.\mathrm{CDCl}_{3}\right): \delta 7.06(\mathrm{~s}, 2 \mathrm{H}), 0.32(\mathrm{~s}, 18 \mathrm{H}) .{ }^{13} \mathrm{C} \mathrm{NMR}\left(67.5 \mathrm{MHz}, \mathrm{CDCl}_{3}\right): \delta 146.28,136.09$, 123.92, 119.37, 82.02, 76.39. $\mathrm{MS}(\mathrm{EI}): \mathrm{m} / \mathrm{e} 516\left(\mathrm{M}^{+}\right)$. Anal. Calcd for $\mathrm{C}_{18} \mathrm{H}_{20} \mathrm{Br}_{2} \mathrm{~S}_{2} \mathrm{Si}_{2}: \mathrm{C}$, 41.86; H, 3.90. Found: C, 41.99; H, 3.93.

1,4-Bis(3-bromo-2-benzothienyl)diacetylene (1f). This compound was prepared from 3bromo-2-benzothienylacetylene essentially in the same manner as described for $\mathbf{1 a}$ in $73 \%$ yield as white solid: mp. 272-273 ${ }^{\circ} \mathrm{C} . \quad{ }^{1} \mathrm{H}$ NMR (500 MHz, $\left.\mathrm{CDCl}_{3}\right): \delta 7.83-7.81(\mathrm{~m}, 2 \mathrm{H})$, 7.77-7.76 (m, 2H), 7.51-7.47 (m, 4H). $\operatorname{HRMS}(\mathrm{EI}): 471.8437\left(\mathrm{M}^{+}\right)$. Calcd for $\mathrm{C}_{20} \mathrm{H}_{8} \mathrm{Br}_{2} \mathrm{~S}_{2}$ : 471.8414 .

\section{A Typical Procedure of the Intramolecular Triple Cyclization.}

Compound 2c. To a solution of compound 1c $(840 \mathrm{mg}, 2.0 \mathrm{mmol})$ in THF $(20 \mathrm{~mL})$ was added a pentane solution of $t$ - BuLi $(1.60 \mathrm{M}, 5.0 \mathrm{~mL}, 8.06 \mathrm{mmol})$ dropwise at $-78{ }^{\circ} \mathrm{C}$. After stirred for $1 \mathrm{~h}$, sulfur $(257 \mathrm{mg}, 8.0 \mathrm{mmol})$ was added as a powder under a stream of argon. The mixture was kept at the same temperature for $5 \mathrm{~min}$ and then was allowed to warm to room temperature with stirring over $2 \mathrm{~h}$. After concentrated under reduced pressure, $1 \mathrm{M}$ sodium hydroxide aqueous solution was added and the mixture was washed with dichloromethane for several times. Potassium ferricyanide(III) was added to the resulting aqueous solution and stirred for $1 \mathrm{~h}$. The mixture was extracted with $\mathrm{CH}_{2} \mathrm{Cl}_{2}$ and the organic layer was washed with water and brine, dried over $\mathrm{MgSO}_{4}$, filtered, and concentrated under reduced pressure. The resulting mixture was subjected to a silica gel column chromatography $\left(1 / 1 \mathrm{CH}_{2} \mathrm{Cl}_{2} /\right.$ hexane $)$ to give $380 \mathrm{mg}(0.98 \mathrm{mmol})$ of $\mathbf{2 c}$ in $49 \%$ yield as purple powders: $\mathrm{mp}$. 203-205 ${ }^{\circ} \mathrm{C} . \quad{ }^{1} \mathrm{H}$ NMR $\left(400 \mathrm{MHz}, \mathrm{CDCl}_{3}\right): \delta 7.66(\mathrm{~d}, J=8.5 \mathrm{~Hz}, 2 \mathrm{H}), 7.29(\mathrm{~d}, J=2.4 \mathrm{~Hz}$, $2 \mathrm{H}), 7.08(\mathrm{dd}, J=8.5,2.4 \mathrm{~Hz}, 2 \mathrm{H}), 3.90(\mathrm{~s}, 6 \mathrm{H}) .{ }^{13} \mathrm{C} \mathrm{NMR}\left(100 \mathrm{MHz}, \mathrm{CDCl}_{3}\right): \delta 158.24$, 139.82, 131.69, 131.03, 123.78, 119.04, 115.32, 105.33, 55.72. Anal. Calcd for $\mathrm{C}_{18} \mathrm{H}_{12} \mathrm{O}_{2} \mathrm{~S}_{4}$ : C, 55.64; H, 3.11. Found: C, 55.67; H, 2.99. 
Compound 2b. $\quad 62 \%$ yield as purple powders: mp. 198.5-199.0 ${ }^{\circ} \mathrm{C} . \quad{ }^{1} \mathrm{H}$ NMR $(500 \mathrm{MHz}$, $\left.\mathrm{CDCl}_{3}\right): \delta 7.89-7.86(\mathrm{~m}, 4 \mathrm{H}), 7.49(\mathrm{td}, J=7.5,1.0 \mathrm{~Hz}, 2 \mathrm{H}), 7.34(\mathrm{td}, J=8.0,1.0 \mathrm{~Hz}, 2 \mathrm{H})$. ${ }^{13} \mathrm{C}$ NMR (100 MHz, $\mathrm{CDCl}_{3}$ ): 140.85, 140.29, 138.74, 126.90, 125.68, 125.58, 125.49, 116.07. MS(EI): m/e $520\left(\mathrm{M}^{+}\right)$. Anal. Calcd for $\mathrm{C}_{16} \mathrm{H}_{8} \mathrm{Se}_{4}: \mathrm{C}, 37.24 ; \mathrm{H}, 1.56$. Found: $\mathrm{C}, 37.31$; H, 1.56.

Compound 2d. $47 \%$ yield as purple powders: mp. 205-206 ${ }^{\circ} \mathrm{C} .{ }^{1} \mathrm{H}$ NMR $(400 \mathrm{MHz}$, $\left.\mathrm{CDCl}_{3}\right): \delta 8.15(\mathrm{~s}, 2 \mathrm{H}), 7.93(\mathrm{~d}, J=8.3 \mathrm{~Hz}, 2 \mathrm{H}), 7.72(\mathrm{~d}, J=8.3 \mathrm{~Hz}, 2 \mathrm{H}) .{ }^{13} \mathrm{C}$ NMR $(100$ $\mathrm{MHz}_{\mathrm{CDCl}}$ ): $\delta \delta 138.98,138.28,136.41,128.16$ (q, $\left.J_{\mathrm{C}-\mathrm{F}}=33 \mathrm{~Hz}\right), 123.95$ (q, $J_{\mathrm{C}-\mathrm{F}}=142 \mathrm{~Hz}$ ), 122.66, $122.40\left(\mathrm{q}, J_{\mathrm{C}-\mathrm{F}}=3.3 \mathrm{~Hz}\right), 121.85,120.30$ (q, $\left.J_{\mathrm{C}-\mathrm{F}}=4.1 \mathrm{~Hz}\right) . \quad \mathrm{MS}(\mathrm{EI}): \mathrm{m} / \mathrm{e} 464\left(\mathrm{M}^{+}\right)$. Anal. Calcd for $\mathrm{C}_{18} \mathrm{H}_{6} \mathrm{~F}_{6} \mathrm{~S}_{4}: \mathrm{C}, 46.54 ; \mathrm{H}, 1.30$. Found: C, 46.57; H, 1.19 .

Compound 2e. $\quad 42 \%$ yield as yellow powders: mp. $193.5-194.0{ }^{\circ} \mathrm{C} . \quad{ }^{1} \mathrm{H}$ NMR $(500 \mathrm{MHz}$, $\left.\mathrm{CDCl}_{3}\right): \delta 7.33(\mathrm{~s}, 2 \mathrm{H}), 0.39(\mathrm{~s}, 18 \mathrm{H}) .{ }^{13} \mathrm{C} \mathrm{NMR}\left(67.5 \mathrm{MHz}, \mathrm{CDCl}_{3}\right): \delta$ 145.38, 143.20, 136.86, 132.80, 125.54, 115.79, -0.25. $\mathrm{MS}(\mathrm{EI}): \mathrm{m} / \mathrm{e} 484\left(\mathrm{M}^{+}\right)$. Anal. Calcd for $\mathrm{C}_{18} \mathrm{H}_{20} \mathrm{~S}_{6} \mathrm{Si}_{2}$ : C, 44.58; H, 4.16. Found: C, 44.35; H, 4.02.

Compound 2f. This compound was purified by recrystallization from $o$-dichlorobenzene. $68 \%$ yield as yellow powders: $\mathrm{mp} .>300{ }^{\circ} \mathrm{C} . \quad{ }^{1} \mathrm{H}$ NMR $\left(500 \mathrm{MHz}, 1 / 9 \mathrm{CDCl}_{3} / \mathrm{CS}_{2}\right): \delta 7.86-$ $7.80(\mathrm{~m}, 4 \mathrm{H}), 7.47-7.38(\mathrm{~m}, 4 \mathrm{H}) .{ }^{13} \mathrm{C}$ NMR spectrum was not able to obtain due to low solubility. $\mathrm{MS}(\mathrm{EI}): \mathrm{m} / \mathrm{e} 440\left(\mathrm{M}^{+}\right)$. Anal. Calcd for $\mathrm{C}_{20} \mathrm{H}_{8} \mathrm{~S}_{6}: \mathrm{C}, 54.51 ; \mathrm{H}, 1.83$. Found: $\mathrm{C}$, 54.47; H, 1.90.

Compoun 2g. This compound was purified by recrystallization from $o$-dichlorobenzene. $47 \%$ yield as yellow brown powders: mp. $>300{ }^{\circ} \mathrm{C} .{ }^{1} \mathrm{H}$ NMR $\left(500 \mathrm{MHz}, 1 / 9 \mathrm{CDCl}_{3} / \mathrm{CS}_{2}\right)$ :

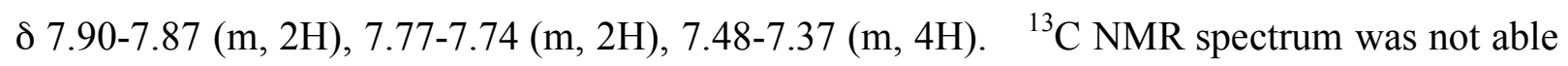
to obtain due to low solubility. HRMS(EI): $629.6729\left(\mathrm{M}^{+}\right)$. Calcd for $\mathrm{C}_{20} \mathrm{H}_{8} \mathrm{~S}_{2} \mathrm{Se}_{4}$ : 629.6736 .

\section{A Trapping Experiment of the Dianionic Intermediate 5 with Benzyl Bromide. 3,3'-} Bis(benzylthio)-2,2'-bi(benzothiophene) (6). To a solution of 1,4-bis $(o$ bromophenyl)diacetylene (1a) $(360 \mathrm{mg}, 1.0 \mathrm{mmol})$ in THF $(10 \mathrm{~mL})$ was added a pentane solution of $t$-BuLi $(1.46 \mathrm{M}, 2.74 \mathrm{~mL}, 4.0 \mathrm{mmol})$ dropwise at $-78{ }^{\circ} \mathrm{C}$. After stirred for $1 \mathrm{~h}$, sulfur $(128 \mathrm{mg}, 4.0 \mathrm{mmol})$ was added as a powder. The mixture was kept at the same temperature for $5 \mathrm{~min}$ and was allowed to warm to room temperature. After stirring for $2 \mathrm{~h}$, benzyl bromide $(262 \mu \mathrm{L}, 2.2 \mathrm{mmol})$ was added. 1M sodium hydroxide solution was added and the mixture was extracted with $\mathrm{CH}_{2} \mathrm{Cl}_{2}$. The organic layer was washed with water and 
brine, dried over $\mathrm{MgSO}_{4}$, filtered, and concentrated under reduced pressure. The resulting mixture was subjected to a silica gel column chromatography $\left(1 / 1 \mathrm{CH}_{2} \mathrm{Cl}_{2} /\right.$ hexane $)$ to give 350 $\mathrm{mg}(0.685 \mathrm{mmol})$ of the bis(benzylthio) compound in $69 \%$ as yellow powders: $\mathrm{mp} .194{ }^{\circ} \mathrm{C}$. ${ }^{1} \mathrm{H}$ NMR $\left(400 \mathrm{MHz}, \mathrm{CDCl}_{3}\right): \delta$ 8.00-7.98 (m, 2H), 7.84-7.82 (m, 2H), 7.45-7.42 (m, 4H), 7.12-7.07 (m, 6H), 7.01-6.99 (m, 4H), $3.90(\mathrm{~s}, 4 \mathrm{H}) .{ }^{13} \mathrm{C} \mathrm{NMR}\left(100 \mathrm{MHz}, \mathrm{CDCl}_{3}\right): \delta 140.29$, 139.67, 138.84, 137.35, 128.83, 128.27, 127.09, 126.58, 125.48, 124.76, 123.82, 122.13, 40.63. HRMS(FAB): $511.0669\left(\mathrm{M}+\mathrm{H}^{+}\right)$. Calcd for $\mathrm{C}_{30} \mathrm{H}_{24} \mathrm{~S}_{2}: 511.0683$.

\section{A Typical Procedure for the Dechalcogenation.}

Compound 3c. The mixture of $2 \mathbf{c}(219 \mathrm{mg}, 0.471 \mathrm{mmol})$ and copper nanopowder (120 mg, $1.89 \mathrm{mmol}$ ) was heated at $240-270{ }^{\circ} \mathrm{C}$ for $10 \mathrm{~min}$ using a sand bath. The reaction mixture was dissolved with $\mathrm{CH}_{2} \mathrm{Cl}_{2}$, and the soluble compounds were collected by filtration with a celite. The filtrate was concentrated under reduced pressure, followed by recrystallization from toluene to give $52.8 \mathrm{mg}(0.148 \mathrm{mmol})$ of $\mathbf{3 c}$ in $58 \%$ yield as a colorless crystal: $\mathrm{mp}$. > $300{ }^{\circ} \mathrm{C} . \quad{ }^{1} \mathrm{H}$ NMR $\left(400 \mathrm{MHz}, \mathrm{CDCl}_{3}\right): \delta 7.74(\mathrm{~d}, J=8.8 \mathrm{~Hz}, 2 \mathrm{H}), 7.37(\mathrm{~d}, J=2.2 \mathrm{~Hz}, 2 \mathrm{H})$, $7.06(\mathrm{dd}, J=8.8,2.2 \mathrm{~Hz}, 2 \mathrm{H}), 3.92(\mathrm{~s}, 6 \mathrm{H}) .{ }^{13} \mathrm{C}$ NMR spectrum was not able to obtain due to low solubility. IR (KBr): 3013 (w), 2955 (w), 2932 (w), 2831 (w), 1600 (s), 1556 (m), 1474 (s), 1435 (s), 1304 (m), 1268 (s), 1226 (s), 1061 (m), 1030 (s), 868 (s), 814 (s) cm cm $^{-1}$ HRMS(EI): $355.9987\left(\mathrm{M}^{+}\right)$. Calcd for $\mathrm{C}_{18} \mathrm{H}_{12} \mathrm{O}_{2} \mathrm{~S}_{3}: 355.9999$.

Compound 3b. $\quad 62 \%$ yield as a colorless crystal: mp. $>300{ }^{\circ} \mathrm{C} . \quad{ }^{1} \mathrm{H}$ NMR $(400 \mathrm{MHz}$, $\left.\mathrm{CDCl}_{3}\right): \delta 7.94(\mathrm{~d}, J=7.1 \mathrm{~Hz}, 2 \mathrm{H}), 7.82(\mathrm{~d}, J=7.1 \mathrm{~Hz}, 2 \mathrm{H}), 7.45$ (t, $\left.J=7.3 \mathrm{~Hz}, 2 \mathrm{H}\right), 7.32$ (t, $J=7.3 \mathrm{~Hz}, 2 \mathrm{H}) .{ }^{13} \mathrm{C} \mathrm{NMR}\left(67.5 \mathrm{MHz}, 4 / 1 \mathrm{CS}_{2} / \mathrm{CDCl}_{3}\right): \delta$ 148.27, 141.97, 137.93, 137.52, 126.60, 125.35, 124.79, 122.85 (against $77.00 \mathrm{ppm}$ of $\mathrm{CDCl}_{3}$ peak as a standard). IR (KBr): 3054 (w), 2924 (w), 2923 (m), 2851 (w), 1444 (s), 1338 (s), 1250 (m), 744 (s), 716 (s) cm cm $^{-1}$ MS(EI): m/e $438\left(\mathrm{M}^{+}\right)$. Anal. Calcd for $\mathrm{C}_{16} \mathrm{H}_{8} \mathrm{Se}_{4}: \mathrm{C}, 43.96 ; \mathrm{H}, 1.84$. Found: $\mathrm{C}, 43.97 ; \mathrm{H}$, 1.79 .

Compound 3d. $\quad 60 \%$ yield as a colorless crystal: mp. $270.4-273.0{ }^{\circ} \mathrm{C}$ (sublimed). $\quad{ }^{1} \mathrm{H}$ NMR $\left(400 \mathrm{MHz}_{\mathrm{CDCl}}\right): \delta 8.21(\mathrm{~s}, 2 \mathrm{H}), 8.00(\mathrm{~d}, J=8.4 \mathrm{~Hz}, 2 \mathrm{H}), 7.72(\mathrm{~d}, J=8.4 \mathrm{~Hz}, 2 \mathrm{H}) .{ }^{13} \mathrm{C}$ $\operatorname{NMR}\left(100 \mathrm{MHz}, 4 / 1 \mathrm{CS}_{2} / \mathrm{CDCl}_{3}\right): \delta 142.09,137.13,135.13,132.43,127.41,127.09,121.94$ (q, $\left.J_{\mathrm{C}-\mathrm{F}}=3.3 \mathrm{~Hz}\right), 121.27\left(\mathrm{q}, J_{\mathrm{C}-\mathrm{F}}=4.1 \mathrm{~Hz}\right.$ ), 120.99 (against $77.00 \mathrm{ppm}$ of $\mathrm{CDCl}_{3}$ peak as a standard). $\mathrm{MS}(\mathrm{EI}): \mathrm{m} / \mathrm{e} 432\left(\mathrm{M}^{+}\right)$. Anal. Calcd for $\mathrm{C}_{18} \mathrm{H}_{6} \mathrm{~F}_{6} \mathrm{~S}_{3}: \mathrm{C}, 50.00 ; \mathrm{H}, 1.40 ; \mathrm{F}, 26.36 ; \mathrm{S}$, 22.25. Found: C, 50.15; H, 1.47; F, 26.21; S, 22.13.

Compound 3e. $\quad 54 \%$ yield as colorless powders: mp. 298.5-300 ${ }^{\circ} \mathrm{C} . \quad{ }^{1} \mathrm{H} \mathrm{NMR}(400 \mathrm{MHz}$, $\left.\mathrm{CDCl}_{3}\right) \delta 7.39(\mathrm{~s}, 2 \mathrm{H}), 0.39(\mathrm{~s}, 18 \mathrm{H}) .{ }^{13} \mathrm{C} \mathrm{NMR}\left(100 \mathrm{MHz}, \mathrm{CDCl}_{3}\right): \delta 142.73,142.23$, 136.66, 134.21, 131.09, 126.70, -0.15. IR (KBr): 3065 (w), 2953 (m), $2923(\mathrm{~m}), 2898(\mathrm{w})$, 


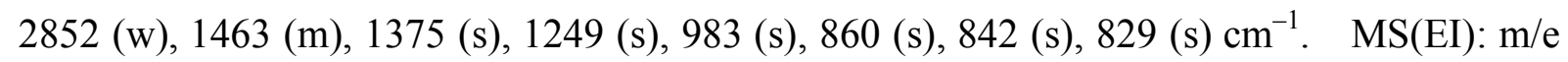
452. $\left(\mathrm{M}^{+}\right)$. Anal. Calcd for $\mathrm{C}_{18} \mathrm{H}_{20} \mathrm{~S}_{5} \mathrm{Si}_{2}$ : C, 47.74; H, 4.45. Found: C, 47.72; H, 4.36.

Compound 3f. $\quad 81 \%$ yield as yellow powders: mp. $>300{ }^{\circ} \mathrm{C}$. $\quad$ IR (KBr): 3048 (w), 2922 (m), 2851 (w), 1467 (m), 1447 (m), 1388 (s), 1335 (m), 1297 (m), 1257 (m), 1129 (m), 737 (s), 719 (s) $\mathrm{cm}^{-1}$. MS(EI): m/e 408. Anal. Calcd for $\mathrm{C}_{20} \mathrm{H}_{8} \mathrm{~S}_{5}: \mathrm{C}, 58.79 ; \mathrm{H}, 1.97$. Found: $\mathrm{C}$, 58.79; $\mathrm{H}, 1.89$. ${ }^{1} \mathrm{H}$ and ${ }^{13} \mathrm{C}$ NMR spectra were not able to obtain due to low solubility.

Compound 3g. $\quad 71 \%$ yield as yellow powders: mp. $>300{ }^{\circ} \mathrm{C}$. IR (KBr): $3046(\mathrm{w}), 2923$ (m), 2850 (w), 1465 (m), 1445 (m), 1357 (s), 1298 (m), 1254 (m), 1127 (m), 739 (s), 718 (s) $\mathrm{cm}^{-1}$. HRMS(EI): $549.7570\left(\mathrm{M}^{+}\right)$. Calcd for $\mathrm{C}_{20} \mathrm{H}_{8} \mathrm{~S}_{2} \mathrm{Se}_{3}:$ 549.7571. Anal. Calcd for $\mathrm{C}_{20} \mathrm{H}_{8} \mathrm{~S}_{2} \mathrm{Se}_{3}$ : C, 43.73; H, 1.47. Found: $\mathrm{C}, 43.75 ; \mathrm{H}, 1.37$. ${ }^{1} \mathrm{H}$ and ${ }^{13} \mathrm{C}$ NMR spectra were not able to obtain due to low solubility.

\section{X-ray Crystal Structure Analysis of $2 b$ and 3a.}

Single crystals of $\mathbf{2 b}$ and 3a suitable for X-ray crystal analysis were obtained by recrystallization from toluene. Intensity data were collected at $173 \mathrm{~K}$ on a Rigaku single

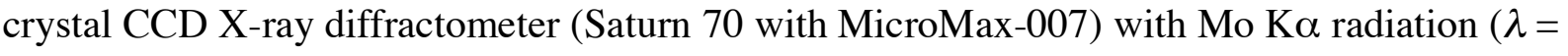
$0.71070 \AA$ A) and graphite monochromator. The crystal data for these compounds are summarized in Table S-1. Their structures were solved by direct methods (SHELXS-97) and refined by the full-matrix least-squares on $F^{2}$ (SHELXL-97). ${ }^{3}$ All non-hydrogen atoms were refined anisotropically and all hydrogen atoms were placed using AFIX instructions.

\section{References}

1. Acheson, R. M.; Lee, G. C. M. J. Chem. Res., Synopses 1986, 380. Haley, M. M.; Bell, M. L.; Brand, S. C.; Kimball, D. B.; Pak, J. J.; Wan, W. B. Tetrahedron Lett. 1997, 38, 7483.

2. Schroth, W.; Hintzsche, E.; Viola, H.; Winkler, R.; Klose, H.; Boese, R.; Kempe, R.; Sieler, J. Chem. Ber. 1994, 127, 401.

3. Sheldrick, G. M. SHELX-97, Program for the Refinement of Crystal Structures; University of Gottingen: Gottingen, Germany, 1997. 
Table S-1. Crystallographic Data for Compounds $\mathbf{2 b}$ and 3a.

\begin{tabular}{lll}
\hline compound & $\mathbf{2 b}$ & $\mathbf{3 a}$ \\
formula & $\mathrm{C}_{16} \mathrm{H}_{8} \mathrm{Se}_{4}$ & $\mathrm{C}_{16} \mathrm{H}_{8} \mathrm{~S}_{3}$ \\
mol weight $\left(\mathrm{g} \mathrm{mol}^{-1}\right)$ & 516.06 & 296.40 \\
cryst dimens $(\mathrm{mm})$ & $0.20 \times 0.20 \times 0.20$ & $0.20 \times 0.20 \times 0.02$ \\
cryst syst & monoclinic & orthorhombic \\
space group & $P 2_{1} / \mathrm{a}$ & $P$ nma \\
a $(\AA)$ & $12.632(8)$ & $7.933(4)$ \\
b $(\AA)$ & $7.864(4)$ & $26.589(15)$ \\
c $(\AA)$ & $14.964(10)$ & $5.906(3)$ \\
$\alpha(\mathrm{deg})$ & & \\
$\beta($ deg$)$ & $101.258(7)$ & \\
$\gamma($ deg$)$ & & \\
$V\left(\AA \AA^{3}\right)$ & $1457.8(15)$ & $1245.9(11)$ \\
$Z$ & 4 & 4 \\
$D_{\text {calcd }}\left(\mathrm{g} \mathrm{cm}{ }^{-3}\right)$ & 2.351 & 1.580 \\
$F(000)$ & 960 & 608 \\
$\mu(\mathrm{Mo} \mathrm{K \alpha})\left(\mathrm{cm}^{-1}\right)$ & 10.050 & 0.573 \\
temp $(\mathrm{K})$ & $173(2)$ & $173(2)$ \\
no. of measd reflns & 8281 & 6907 \\
no. of ind reflns & $2467(\mathrm{I}>2 \sigma(\mathrm{I}))$ & $1110(\mathrm{I}>2 \sigma(\mathrm{I}))$ \\
goodness of fit & 1.085 & 1.218 \\
final $R$ indices & & \\
$R 1$ & 0.0510 & 0.0417 \\
$w R 2(I>2 \sigma(I))$ & 0.1309 & 0.0913 \\
& & \\
\hline
\end{tabular}


Table S-2. UV-vis Absorption Spectral Data for Fused 1,2-Dichalcogenins 2 and Heteroacenes $\mathbf{3}$

\begin{tabular}{ll} 
cmpd & $\lambda_{\max } / \mathrm{nm}(\log \varepsilon)^{a}$ \\
\hline $\mathbf{2 a}$ & $468(3.41), 338(4.25)$ \\
$\mathbf{2 b}$ & $498(2.12), 366(4.20)$ \\
$\mathbf{2 c}$ & $473(3.56), 357(4.46)$ \\
$\mathbf{2 d}$ & $471(3.49), 334(4.36)$ \\
$\mathbf{2 e}$ & $473(3.81), 347(4.40)$ \\
$\mathbf{2 f}$ & $480(3.99), 343(4.42)$ \\
$\mathbf{2 g}$ & $516(3.51), 379(4.18)$ \\
$\mathbf{3 a}$ & $344(4.51), 327(4.56)$ \\
$\mathbf{3 b}$ & $356(4.56), 340(4.59)$ \\
$\mathbf{3 c}$ & $356(4.43), 340(4.41), 321(4.20)$ \\
$\mathbf{3 d}$ & $350(4.59), 332(4.62)$ \\
$\mathbf{3 e}$ & $370(4.65), 354(4.69), 337(4.46), 323(4.42)$ \\
$\mathbf{3 f}^{b}$ & $399(3.98), 378(3.93)$ \\
$\mathbf{3 g}^{b}$ & $418(3.08), 394(3.04)$ \\
\hline
\end{tabular}

${ }^{a}$ In THF, unless otherwise stated. ${ }^{b}$ In $\mathrm{CS}_{2}$. 